\title{
Particle swarm optimization with fractional-order velocity
}

\author{
E.J. Solteiro Pires, J.A. Tenreiro Machado, P.B. de Moura Oliveira, J. Boaventura Cunha, Luís Mendes
}

\begin{abstract}
This paper proposes a novel method for controlling the convergence rate of a particle swarm optimization algorithm using fractional calculus (FC) concepts. The optimization is tested for several wellknown functions and the relationship between the fractional order velocity and the convergence of the algorithm is observed. The FC demonstrates a potential for interpreting evolution of the algorithm and to control its convergence.
\end{abstract}

Keywords Particle swarm optimization · Fractional calculus

\section{Introduction}

In the last decade, particle swarming optimization (PSO) has been applied in a plethora of fields such as social modelling, computer graphics, simulation and animation of natural flocks or swarms, pattern recognition, color image quantisation, and computational biology [1]. PSO has seduced considerable interest from the natural computing research, where important work has been enforced in the study of its convergence.

Fractional Calculus (FC) is a natural extension of the classical mathematics. In fact, since the beginning of theory of differential and integral calculus, several mathematicians investigated the calculation of noninteger order derivatives and integrals. Nevertheless, the application of FC has been scarce until recently, but the recent scientific advances motivated a renewed interest in this field.

Bearing these ideas in mind, this work uses a fractional derivative to control the convergence rate of the PSO. The article is organized as follows. Section 2 introduces the FC. Section 3 presents the PSO and its working principles. Based on this formulation, Sect. 4 generalizes the PSO to a fractional order. Section 5 presents the results for the PSO with fractional velocity. Finally, Sect. 6 outlines the main conclusions.

\section{Fractional calculus}

FC goes back to the beginning of the theory of differential calculus. Nevertheless, the application of FC 
just emerged in the last two decades, due to the progresses in the area of nonlinear and complex systems that revealed subtle relationships with the FC concepts. In the field of dynamical systems theory, some work has been carried out, but the proposed models and algorithms are still in a preliminary stage of establishment.

The fundamentals aspects of FC theory are addressed in [2-5]. Concerning FC applications research efforts can be mentioned in the area of viscoelasticity, chaos, fractals, biology, electronics, signal processing, diffusion, wave propagation, percolation, modeling, physics, control, and irreversibility [6-14].

FC is a branch of mathematical analysis that extends to real or even complex numbers the order of the differential and integral operators. Since its foundation, the generalization of the concept of derivative and integral to a noninteger order $\alpha$ has been the subject of distinct approaches. Due to this reason there are several alternative definitions of fractional derivatives. For example, the Laplace definition of a derivative of fractional order $\alpha \in \mathrm{C}$ of the signal $x(t), D^{\alpha}[x(t)]$, is a 'direct' generalization of the classical integer-order scheme yielding:

$$
\mathcal{L}\left\{D^{\alpha}[x(t)]\right\}=s^{\alpha} X(s)
$$

for zero initial conditions. This means that frequencybased analysis methods have a straightforward adaptation. An alternative approach, based on the concept of fractional differential, is the Grünwald-Letnikovdefinition given by the equation:

$$
D^{\alpha}[x(t)]=\lim _{h \rightarrow 0}\left[\frac{1}{h^{\alpha}} \sum_{k=0}^{+\infty} \frac{(-1)^{k} \Gamma(\alpha+1) x(t-k h)}{\Gamma(k+1) \Gamma(\alpha-k+1)}\right]
$$

An important property revealed by expression (2) is that while an integer-order derivative just implies a finite series, the fractional-order derivative requires an infinite number of terms. Therefore, integer derivatives are "local" operators in opposition with fractional derivatives which have, implicitly, a "memory" of all past events.

Often, in discrete time implementations expression (3) is approximate by

$$
D^{\alpha}[x(t)]=\frac{1}{T^{\alpha}} \sum_{k=0}^{r} \frac{(-1)^{k} \Gamma(\alpha+1) x(t-k T)}{\Gamma(k+1) \Gamma(\alpha-k+1)}
$$

where $T$ is the sampling period and $r$ is the truncation order.

The characteristics revealed by fractional-order models make this mathematical tool well suited to describe phenomena such as irreversibility and chaos because of its inherent memory property. In this line of thought, the propagation of perturbations and the appearance of long-term dynamic phenomena in a population of individuals subjected to an evolutionary process configure a case where FC tools fit adequately [15].

\section{Particle swarm optimization algorithm}

Evolutionary algorithms have been successfully applied to solve many complex optimization engineering problems. Together with genetic algorithms, the PSO algorithm, proposed by Kennedy and Eberhart [16], has achieved considerable success in solving optimization problems.

The PSO algorithm was proposed originally in [16]. This optimization technique is inspired in the way swarms behave and its elements move in a synchronized way, both as a defensive tactic and for searching food. An analogy is established between a particle and a swarm element. The particle movement is characterized by two vectors, representing its current position $x$ and velocity $v$. Since 1995, many techniques were proposed to refine and/or complement the original canonical PSO algorithm, namely by analyzing the tuning parameters [17] and by considering hybridization with other evolutionary techniques [18].

In literature, some work embedding FC and PSO algorithms can be found. Pires et al. [19] studies the fractional dynamics during the evolution of a PSO. Reis et al. [20] proposes a PSO, for logic and circuit design, where a proportional-derivative fitness function is implemented to guide the optimization.

Algorithm 1 illustrates a standard PSO algorithm. The basic algorithm begins by initializing the swarm randomly in the search space. As it can be seen in the pseudo-code, were $t$ and $t+1$ represent two consecutive iterations, the position $x$ of each particle is updated during the iterations by adding a new velocity $v$. This velocity is evaluated by summing an increment to the previous velocity value. The increment is a function of two components representing the cognitive and the social knowledges. 


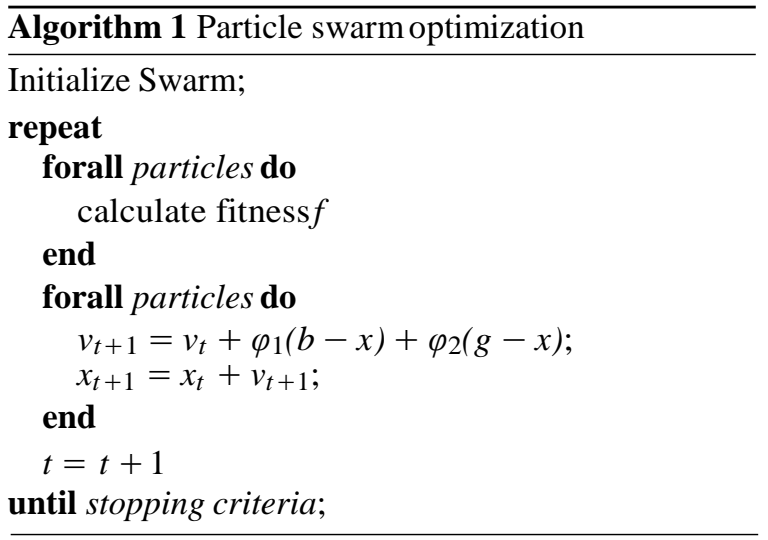

The cognitive knowledge of each particle is included by evaluating the difference between its best position found so far $b$ and the current position $x$. On the other side, the social knowledge, of each particle, is incorporated through the difference between the best swarm global position achieved so far $g$ and its current position $x$. The cognitive and the social knowledge factors are multiplied by randomly uniformly generated terms $\varphi_{1}$ and $\varphi_{2}$, respectively.

PSO is a optimization algorithm that proves to be efficient, robust and simple. However, if no care is taken the velocities may attain large values, particularly when particles are far away from local and global bests. Some approaches were carried out in order to eliminate this drawback. Eberhat et al. [21] proposed a clamping function (4) to limit the velocity, through the expression:

$$
\begin{aligned}
& v_{i j}(t+1)= \begin{cases}v_{i j}^{\prime}(t+1) & \text { if } v_{i j}^{\prime}(t+1)<V_{\max } j \\
V_{\max j} & \text { if } v_{i j}^{\prime}(t+1) \geq V_{\max } j\end{cases} \\
& \text { where } v_{i j}^{\prime}(t+1) \quad \text { results from } v_{i j}^{\prime}(t+1)= \\
& v_{i j}(t)+\phi_{1}(b-x)+\phi_{2}(g-x) \text { for the parameter } j \\
& \text { of particle } i \text { at iteration } t+1 .
\end{aligned}
$$

Later, a constant, the inertia weight, was introduced [17] to control the velocity from exploding (5). The inertia weight $\omega$ is very important to ensure convergence behavior over evolution by adopting the equation:

$$
v_{t+1}=\omega v_{t}+\phi_{1}(b-x)+\phi_{2}(g-x)
$$

Some empirical and theoretical studies were made to determine the best inertia value [22] in order to obtain better PSO behavior. Later the same authors [23] indicated that initializing $\omega$ to 0.9 and reducing it lin- early to 0.4 , during the evolution, would allow initial exploration toward a local search as iterations follow. An alternative technique, to prevent velocity explosion, proposed by Clerc and Kennedy [24] uses a constriction $\chi$ in place of clamping the velocity:

$$
\begin{aligned}
& v_{t+1}=\chi\left(v_{t}+\phi_{1} \beta_{1}(b-x)+\phi_{2} \beta_{2}(g-x)\right) \\
& \chi=\frac{2}{\left|2-\phi-\sqrt{\phi^{2}-4 \phi}\right|} \\
& \text { where } \phi=\phi_{1}+\phi_{2} \text {, and } \phi>4 .
\end{aligned}
$$

\section{PSO with fractional velocity}

In this section, a new method to control the PSO algorithm is introduced. Initially, the original velocity equation (7) is rearranged in order to modify the order of the velocity derivative, namely

$$
v_{t+1}=v_{t}+\phi_{1}(b-x)+\phi_{2}(g-x)
$$

This expression can be rewritten as:

$$
v_{t+1}-v_{t}=\phi_{1}(b-x)+\phi_{2}(g-x)
$$

The left side $v_{t+1}-v_{t}$ is the discrete version of the derivative of order $\alpha=1$ (assuming $T=1$ ), leading to the following expression:

$$
D^{\alpha}\left[v_{t+1}\right]=\phi_{1}(b-x)+\phi_{2}(g-x)
$$

The order of the velocity derivative can be generalized to a real number $0 \leq \alpha \leq 1$, if the FC perspective is considered, leading to a smoother variation and a longer memory effect. In order to study the behavior of this new PSO strategy, a set of simulations are carried on testing values of $\alpha$ ranging from $\alpha=0$ up to $\alpha=1$, with increments of $x=0.1$. Therefore, (9) can be written as (10) considering the first $r=4$ terms of differential derivative given by (3), yielding

$$
\begin{aligned}
v_{t+1} & -\alpha v_{t}-\frac{1}{2} \alpha v_{t-1}-\frac{1}{6} \alpha(1-\alpha) v_{t-2} \\
& -\frac{1}{24} \alpha(1-\alpha)(2-\alpha) v_{t-3} \\
= & \phi_{1}(b-x)+\phi_{2}(g-x)
\end{aligned}
$$


or

$$
\begin{aligned}
v_{t+1}= & \alpha v_{t}+\frac{1}{2} \alpha v_{t-1}+\frac{1}{6} \alpha(1-\alpha) v_{t-2} \\
& +\frac{1}{24} \alpha(1-\alpha)(2-\alpha) v_{t-3} \\
& +\phi_{1}(b-x)+\phi_{2}(g-x)
\end{aligned}
$$

Larger values of $r$ were tested leading to results of the same type.

\section{Test functions}

This section introduces the optimization functions that are adopted during the tests of PSO with fractional velocity update. The objective function consists in minimizing several well known functions namely: Bohachevsky 1, Colville, Drop wave, Easom, and Rastrigin represented in expressions (12-16), respectively [22]:

Bohachevsky 1 function:

$$
\begin{aligned}
f_{1}(x)= & x_{1}^{2}+2 x_{2}^{2}-0.3 \cos \left(3 \pi x_{1}\right) \\
& -0.4 \cos \left(4 \pi x_{2}\right)+0.7
\end{aligned}
$$

with $x_{i} \in[-50,50], i=\{1,2\}$ and $f^{*}(x)=0.0$.

\section{Colville function:}

$$
\begin{aligned}
f_{2}(x)= & 100\left(x_{2}-x_{1}^{2}\right)^{2}+\left(1-x_{1}\right)^{2}+90\left(x_{4}-x_{3}^{2}\right)^{2} \\
& +\left(1-x_{3}\right)^{2}+10.1\left(\left(x_{2}-1\right)^{2}+\left(x_{4}-1\right)^{2}\right) \\
& +19.8\left(x_{2}-1\right)\left(x_{4}-1\right)
\end{aligned}
$$

with $x_{i} \in[-10,10], i=\{1,2,3,4\}$ and $f^{*}(x)=0.0$.

$$
f_{3}(x)=-\frac{1+\cos \left(12 \sqrt{x_{1}^{2}+x_{2}^{2}}\right)}{0.5\left(x_{1}^{2}+x_{2}^{2}\right)+2}
$$

with $x_{i} \in[-10,10], i=\{1,2\}$ and $f^{*}(x)=0.0$.

\section{Easom function:}

$$
\begin{aligned}
& f_{4}(x)=-\cos \left(x_{1}\right) \cos \left(x_{2}\right) \mathrm{e}^{-\left(x_{1}-\pi\right)^{2}-\left(x_{2}-\pi\right)^{2}} \\
& \text { with } x_{1}, x_{2} \in[-100,100] \text { and } f^{*}(x)=-1.0 .
\end{aligned}
$$

Rastrigin function:

$$
\begin{aligned}
& f_{5}(x)=\sum_{j=1}^{n}\left(x_{j}^{2}-10 \cos \left(2 \pi x_{j}\right)+10\right) \\
& \text { with } n=30, x_{i} \in[-5.12,5.12], i=\{1, \ldots, n\} \text { and } \\
& f^{*}(x)=0.0 .
\end{aligned}
$$

These functions have $n$ parameters, $i=\{1, \ldots, n\}$ and their global optimum value is $f^{*}$. The algorithm adopts a real encoding scheme.

\section{Simulation results}

To study the influence of the fractional velocity in the algorithm, several tests are now developed. A 10population size PSO is executed during a period of 200 iterations with $\left\{\varphi_{1}, \varphi_{2}\right\} \sim U[0,1]$. The fitness evolution of the best global particle is taken as the system output.

Since PSO is a stochastic algorithm, every time it is executed it leads to a different trajectory convergence. Therefore a test group of 2ak simulations was consideach value in the set $\alpha=\{0,0.1, \ldots, 1\}$. In Figs. 1-5, the results can be seen for the adopted optimization functions $f_{j}, j=\{1, \ldots, 5\}$.

From the figures, it can be verified that the convergence of the algorithm depends directly upon the fractional order $\alpha$. With exception of the Easom function, as $\alpha$ value increases the speed of convergence of the

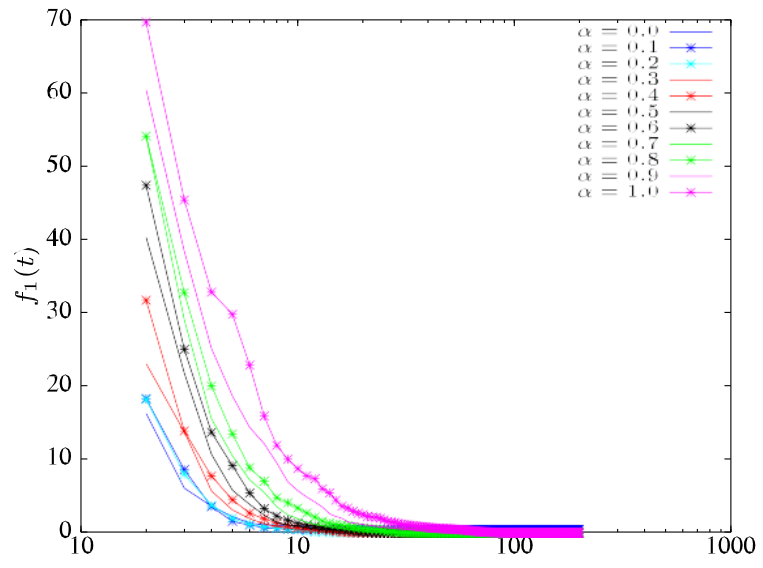

Fig. 1 Evolution of the Bohachevsky 1 function for $\alpha=\{0, \ldots, 1\}$, with best value of $\alpha=0.19$ 


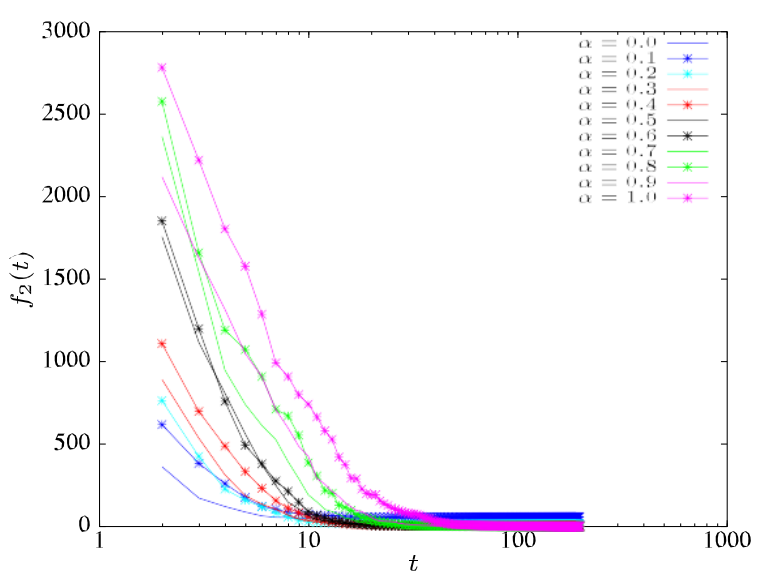

Fig. 2 Evolution of the Colville function for $\alpha=\{0, \ldots, 1\}$, with best value of $\alpha=0.84$

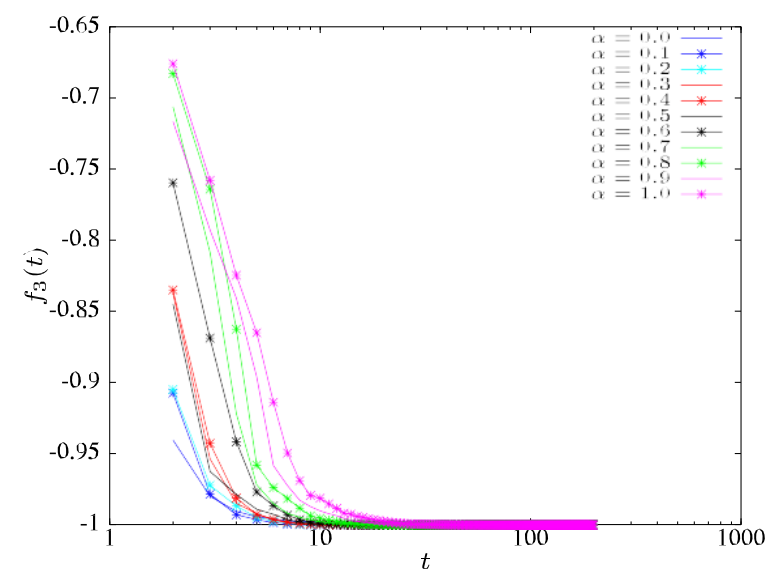

Fig. 3 Evolution of the Drop wave function for $\alpha=\{0, \ldots, 1\}$, with best value of $\alpha=0.18$

PSO decreases. However, with low values, the probability that the population can be trapped in a local optimum becomes higher. For the Easom function, the fastest convergence is obtained when $\alpha=0.33$. As the $\alpha$ differs from this value, the convergency is slower. In both cases, convergence varies in a consistent form.

Additional experiments were carried out for the Ackley, De Jong, and Rosenbrock functions. In these experiments, the convergence behavior was similar with the Bohachevsky 1, Colville, Drop wave, and Rastrigin functions.

In all functions, faster optimization convergence was obtained for values of $\alpha$ in the range [0.18, 0.84]. The value drawn varies according with the optimization function used. In all cases, fractional PSO out-

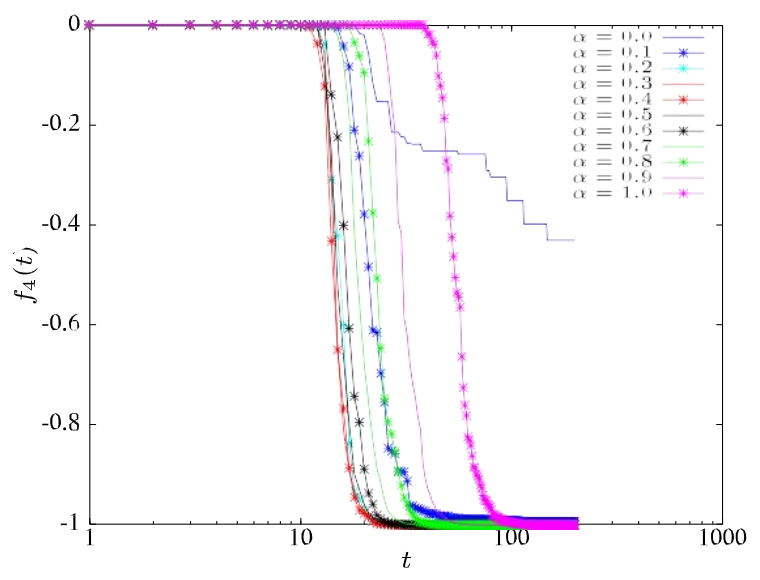

Fig. 4 Evolution of the Easom function for $\alpha=\{0, \ldots, 1\}$, with best value of $\alpha=0.33$

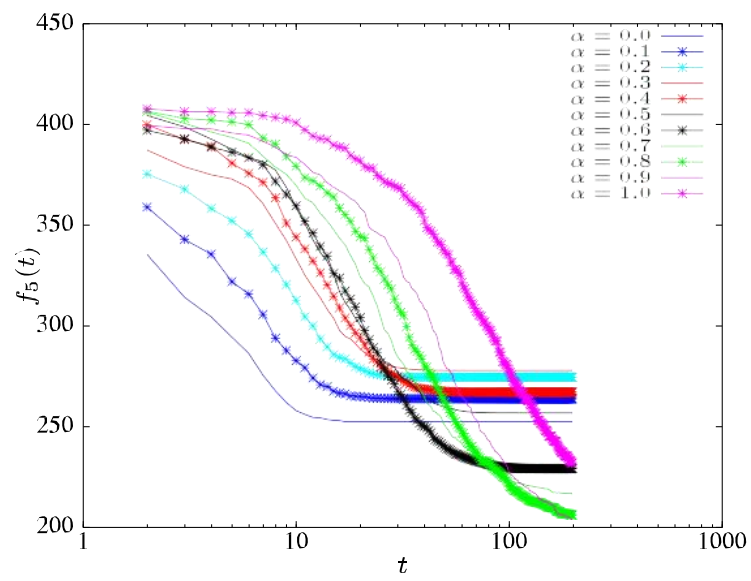

Fig. 5 Evolution of the Rastrigin function for $\alpha=\{0, \ldots, 1\}$, with best value of $\alpha=0.81$

performs standard PSO in the convergence perspective point of view.

In the sequel, several experiments are developed where the fractional-order of the velocity derivative, $\alpha$, varies linearly during the swarm evolution. The results are compared when adopting the alternative scheme of varying the inertia factor $\omega$.

In this line of thought, the value of $\alpha$ varies linearly from $0.9(t=0)$ up to $0.3(t=200)$ according to the expression:

$$
\alpha(t)=0.9-0.6 \frac{t}{200}, \quad t=0,1, \ldots, 200
$$

Figures 6-10 show the time evolution of the best swarm element fitness. Additionally in the figures, are 


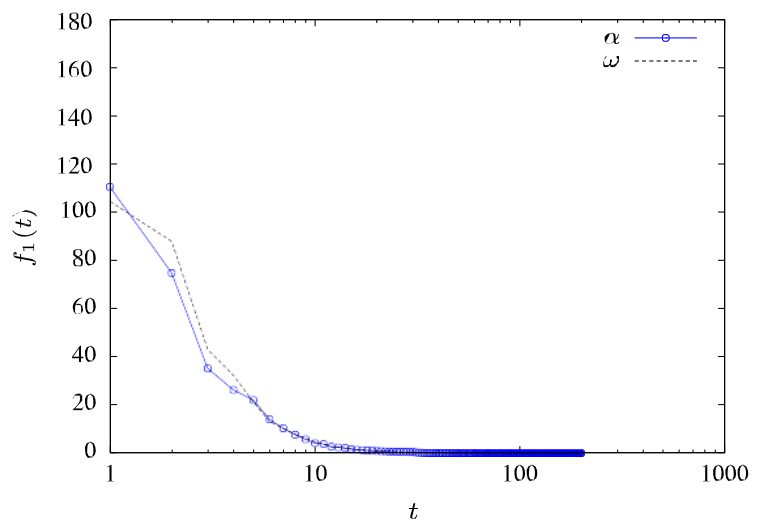

Fig. 6 Evolution of the Bohachevsky 1 function, with variable $\alpha$ and $\omega$

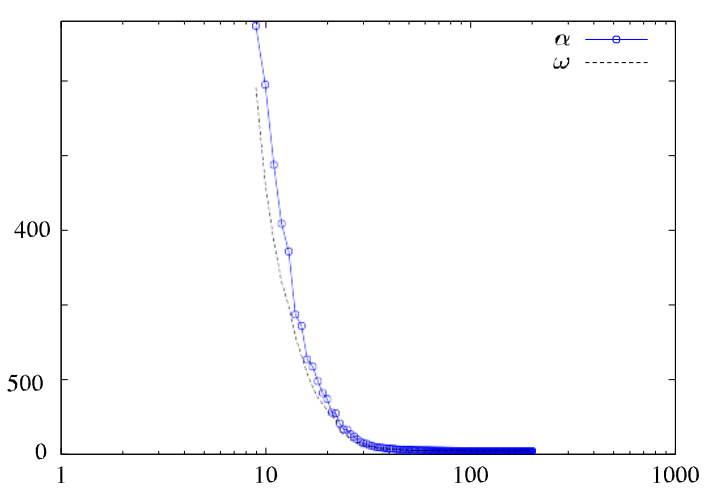

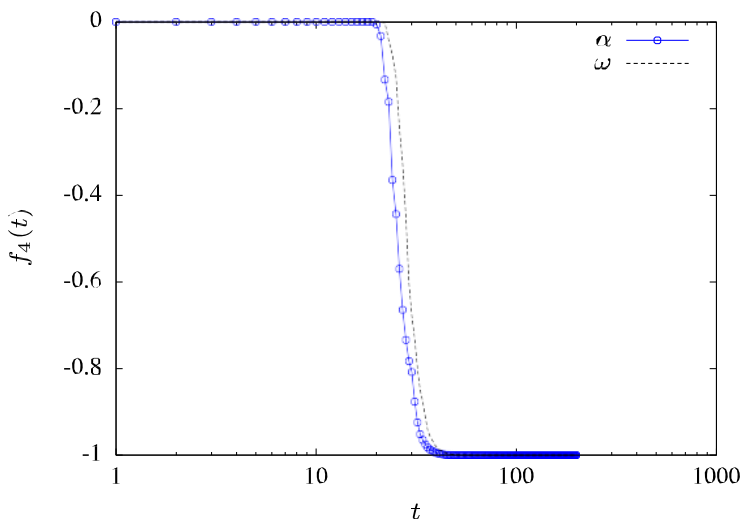

Fig. 9 Evolution of the Easom function, with variable $\alpha$ and $\omega$

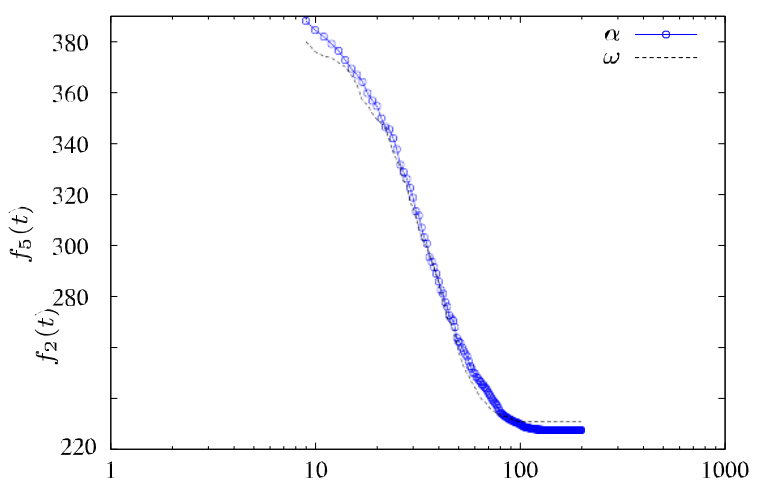

Fig. 7 Evolution of the Colville function, with variable $\alpha$ and $\omega$

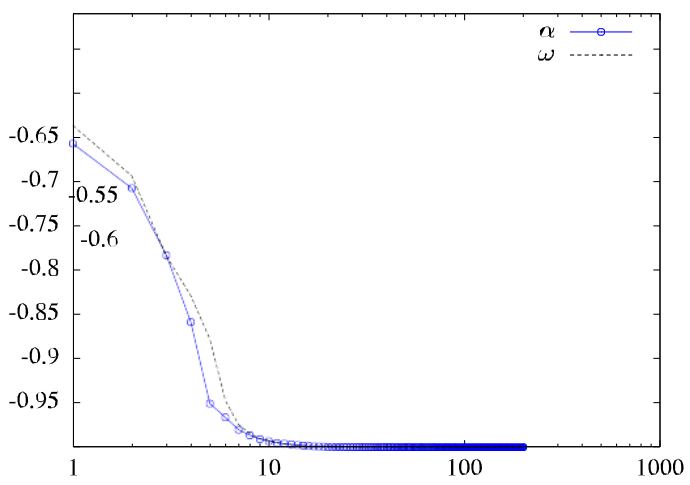


$\underset{\underbrace{\infty}_{\infty}}{\oplus^{\infty}}$

Fig. 10 Evolution of the Rastrigin function, with variable $\alpha$ and $\omega$

depicted the results obtained with PSO when (5) is used and $\omega$ varies also linearly:

$\omega(t)=0.9-0.6_{200}^{t}, \quad t=0,1, \ldots, 200$

Comparing the tests results, it can be observed that both simulations reveal similar behavior. However, the use of fractional velocity contributes to an alternative understanding of the convergence dynamics. In fact, while the decrease in $\omega$ in (18) represents only higher accelerations and higher volatility, the decrease in $\alpha$ in (17) indicates the presence of a long term behavior,

Fig. 8 Evolution of the Drop wave function, with variable $\alpha$ and $\omega$ by incorporating a more significant amount of past information, which in not attained for the case of $\alpha=1$. Bearing this fact in mind, it is verified that FC represents a natural tool which permits to model nonlocal phenomena in time evolution, and points to future promising developments based on a new viewpoint. 


\section{Conclusions}

FC is a well-developed mathematical tool which permits to understand the local and global velocity characteristics of the PSO behavior.

The fractional order velocity was analyzed. It was shown that it directly influences the speed of convergence of the algorithm. Moreover, the results are consistent representing an important step to understand the relationship between velocity memory and the convergence behavior.

The FC concepts open new perspectives toward the development of more efficient swarm optimization.

\section{References}

1. Banks, A., Vincent, J., Anyakoha, C.: A review of particle swarm optimization. ii: Hybridisation, combinatorial, multicriteria and constrained optimization, and indicative applications. Nat. Comput. 7(1), 109-124 (2008)

2. Gement, A.: On fractional differentials. Proc. Philos. Mag. 25, 540-549 (1938)

3. Oustaloup, A.: La Commande CRONE: Commande Robuste d'Ordre Non Intier. Hermes, Paris (1991)

4. Méhauté, A.L.: Fractal Geometries: Theory and Applications. Penton Press, Cleveland (1991)

5. Podlubny, I.: Fractional Differential Equations. Academic Press, San Diego (1999)

6. Tenreiro Machado, J.A.: Analysis and design of fractionalorder digital control systems. J. Syst. Anal.-Model. Simul. 27, 107-122 (1997)

7. Tenreiro Machado, J.A.: System modeling and control through fractional-order algorithms. FCAA-J. Fractional Calc. Appl. Anal. 4, 47-66 (2001)

8. Vinagre, B.M., Petras, I., Podlubny, I., Chen, Y.Q.: Using fractional order adjustment rules and fractional order reference models in model-reference adaptive control. Nonlinear Dyn. 1-4(29), 269-279 (2002)

9. Torvik, P.J., Bagley, R.L.: On the appearance of the fractional derivative in the behaviour of real materials. ASME J. Appl. Mech. 51, 294-298 (1984)

10. Westerlund, S.: Dead Matter Has Memory! Causal Consulting. Kalmar, Sweden (2002)

11. Herzallah, M.A.E., Baleanu, D.: Fractional-order EulerLagrange equations and formulation of Hamiltonian equations. Nonlinear Dyn. 58(1-2), 385-391 (2009)

12. Rabei, E.M., Altarazi, I.M.A., Muslih, S.I., Baleanu, D.: Fractional WKB approximation. Nonlinear Dyn. 57(1-2), 171-175 (2009)
13. Tarasov, V.E., Zaslavsky, G.M.: Fokker-Planck equation with fractional coordinate derivatives. Physica A, Stat. Mech. Appl. 387(26), 6505-6512 (2008)

14. Magin, R., Feng, X., Baleanu, D.: Solving the fractional order Bloch equation. Concepts Magn. Reson. 34A(1), 16 23 (2009)

15. Solteiro Pires, E.J., de Moura Oliveira, P.B., Tenreiro Machado, J.A., Jesus, I.S.: Fractional order dynamics in a particle swarm optimization algorithm. In: Seventh International Conference on Intelligent Systems Design and Applications, ISDA 2007, Washington, DC, USA, pp. 703-710. IEEE Computer Society, Los Alamitos (2007)

16. Kennedy, J., Eberhart, R.C.: Particle swarm optimization. In: Proceedings of the 1995 IEEE International Conference on Neural Networks, Perth, Australia, vol. 4, pp. 19421948. IEEE Service Center, Piscataway (1995)

17. Shi, Y., Eberhart, R.: A modified particle swarm optimizer. In: Evolutionary Computation Proceedings, 1998. IEEE World Congress on Computational Intelligence, The 1998 IEEE International Conference on, Anchorage, Alaska, pp. 69-73 (1998)

18. Løvbjerg, M., Rasmussen, T.K., Krink, T.: Hybrid particle swarm optimiser with breeding and subpopulations. In: Spector, L., Goodman, E.D., Wu, A., Langdon, W., Voigt, H.M., Gen, M., Sen, S., Dorigo, M., Pezeshk, S., Garzon, M.H., Burke, E. (eds.) Proceedings of the Genetic and Evolutionary Computation Conference (GECCO-2001), San Francisco, California, USA (7-11 July), pp. 469-476. Morgan Kaufmann, San Mateo (2001)

19. Solteiro Pires, E.J., Tenreiro Machado, J.A., de Moura Oliveira, P.B., Reis, C.: Fractional dynamics in particle swarm optimization. In: ISIC. IEEE International Conference on Systems, Man and Cybernetics, Montreal, Que. (7-10 Oct. 2007), pp. 1958-1962

20. Reis, C., Machado, J., Galhano, A., Cunha, J.: Circuit synthesis using particle swarm optimization. In: IEEE International Conference on Computational Cybernetics (ICCC 2006) (Aug. 2006), pp. 1-6

21. Eberhart, R., Simpson, P., Dobbins, R.: Computational Intelligence PC Tools. Academic Press, San Diego (1996)

22. den Bergh, F.V., Engelbrecht, A.P.: A study of particle swarm optimization particle trajectories. Inf. Sci. 176(8), 937-971 (2006)

23. Eberhart, R., Shi, Y.: Comparing inertia weights and constriction factors in particle swarm optimization. In: Proceedings of the 2000 Congress on Evolutionary Computation, Washington, DC, vol. 1, pp. 84-88 (2000)

24. Clerc, M., Kennedy, J.: The particle swarm-explosion, stability, and convergence in a multidimensional complex space. IEEE Trans. Evol. Comput. 6(1), 58-73(2002) 